\title{
ORIGINAL ARTICLE Sudden versus gradual pressure wean from Nasal CPAP in preterm infants: a randomized controlled trial
}

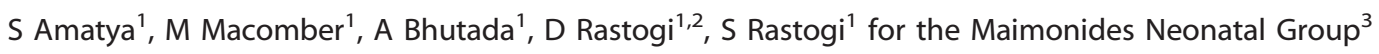

OBJECTIVE: In preterm infants, nasal continuous positive airway pressure (NCPAP) is widely used for treatment of respiratory distress syndrome. However, the strategies for successfully weaning infants off NCPAP are still not well defined and there remains considerable variation between the methods. The objective of this study is to determine whether gradual weaning of NCPAP pressure is more successful than sudden weaning off NCPAP to room air.

STUDY DESIGN: A randomized controlled trial was conducted in a level 3 neonatal intensive care unit on 70 preterm neonates who were born between 26 and 32 weeks gestation and required NCPAP for at least $48 \mathrm{~h}$. When infants were stable on NCPAP at 0.21 $\mathrm{FiO}_{2}$ and $5 \mathrm{~cm} \mathrm{H}_{2} \mathrm{O}$ positive end expiratory pressure, neonates were randomized to the gradual wean group (reduction in pressure by $1 \mathrm{~cm}$ every $8 \mathrm{~h}$ until $3 \mathrm{~cm} \mathrm{H} 0$ was reached) or to sudden wean group (one time NCPAP removal to room air). The primary outcome was a success at the first trial to wean to room air. Secondary outcomes were a number of trials, and weight and postmenstrual age (PMA) at the time of successful wean. Total number of days on NCPAP and length of stay (LOS) in the hospital were also compared between the groups.

RESULTS: Of the 70 infants included in the study, 35 were randomized to sudden group and 33 infants to gradual group ( 2 excluded for protocol deviation). In sudden and gradual groups, 14 and 22 infants, respectively, were weaned successfully in the first attempt $(P=0.03)$. The infants were successfully weaned at $32.7 \pm 1.7$ weeks versus $33.1 \pm 2.4$ weeks $(P=0.39) P M A$ and at a weight of $1651 \pm 290 \mathrm{~g}$ versus $1589 \pm 398 \mathrm{~g}(P=0.46)$ in the sudden and gradual groups, respectively. The total number of days on NCPAP was $27 \pm 19$ days versus $32 \pm 24$ days $(P=0.38)$ and LOS was $63 \pm 25$ days versus $63 \pm 22$ days $(P=0.99)$ in the sudden and gradual groups, respectively.

CONCLUSIONS: Gradual weaning method was more successful as compared to sudden weaning method in the initial trial off NCPAP. There was no difference in the PMA, weight at the time of successful wean, total days on NCPAP and LOS between the two groups.

Journal of Perinatology (2017) 37, 662-667; doi:10.1038/jp.2017.10; published online 23 February 2017

\section{INTRODUCTION}

Nasal continuous positive airway pressure (NCPAP) is widely used for the treatment of respiratory distress syndrome in preterm infants $^{1}$ and is associated with lower incidence of intubation and its related complications as compared with other modes of ventilation. ${ }^{2-6}$ Early use of NCPAP in preterm infants is associated with decreased pulmonary ${ }^{7,8}$ and non-pulmonary ${ }^{9-11}$ morbidities that are associated with intubation and ventilation. It has also been shown to improve lung growth. ${ }^{12}$ Although there are risks associated with NCPAP including nasal trauma, ${ }^{13}$ increased incidence of pneumothorax ${ }^{2}$ and possible association with intraventricular ventricular hemorrhage, ${ }^{14,15}$ early weaning off NCPAP can lead to atelectasis, apnea and bradycardia, leading to prolonged use of NCPAP, or possibly intubation with subsequent mechanical ventilation and prolonged oxygen use. ${ }^{1}$ These findings suggest that weaning off NCPAP should be planned appropriately. However, there is a lack of consensus regarding the optimal method of weaning NCPAP and timing are often implemented on ad-hoc basis. ${ }^{16}$

In recent years, investigations into the optimal method and timing of weaning off NCPAP ${ }^{16,17}$ have highlighted the considerable variation that exists in the methods and the timing used for weaning of NCPAP. The varied weaning methods that have been used include sudden removal of NCPAP, gradual increase in time off NCPAP, gradual reduction of NCPAP pressure with or without oxygen supplementation, transition to high-flow nasal cannula (NC) or a combination of all of these methods. ${ }^{18-23}$

We had reported factors associated with successful wean from NCPAP in preterm infants and the clinical criteria for defining readiness to wean, successful wean and failure of wean. ${ }^{24}$ However, there is no consensus on the optimal method of weaning. Although we have previously found no difference between sudden wean of NCPAP and a gradual cycling of time off NCPAP, ${ }^{23}$ others have shown that sudden weaning may be associated with a shorter weaning time. ${ }^{22}$ In the ceasing CPAP at standard criteria (CICADA) study, once stability criteria was met, NCPAP was taken off in a sudden manner and this led to shorter NCPAP weaning time, NCPAP and oxygen duration, and lower incidence of broncho-pulmonary dysplasia, as compared with gradual cycling off NCPAP with or without NC. ${ }^{22,25}$ Conversely, Singh et al. ${ }^{19}$ reported a gradual decrease in pressure of NCPAP to be better than a gradual time off NCPAP in terms of successful wean and duration of NCPAP. Based on this conflicting literature on the NCPAP wean methods, we hypothesized that gradual

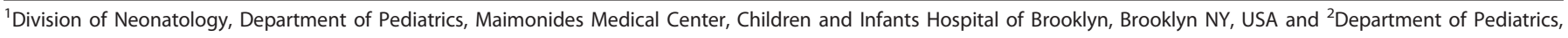

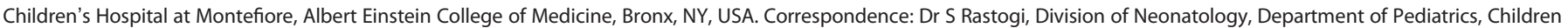
and Infants Hospital of Brooklyn, Maimonides Medical Center, 1048 Tenth Avenue, F113, Brooklyn, NY 11219, USA.

E-mail: srastogi@maimonidesmed.org

${ }^{3}$ Members of Maimonides Neonatal Group are listed above references.

Received 25 October 2016; revised 3 January 2017; accepted 17 January 2017; published online 23 February 2017 
pressure wean as compared with sudden wean would be more successful in the first attempt in trialing off NCPAP. We also hypothesized that gradual pressure weaning would also be associated with shorter time on NCPAP and shorter length of stay (LOS) in the hospital.

\section{METHODS}

Study design

A prospective randomized controlled trial was conducted on preterm infants born at 26 weeks to 32 weeks of gestation at Maimonides Infant and Children's Hospital between October 2013 and November 2015. The study was approved by the Institutional Review Board at Maimonides Medical Center and was conducted in compliance with the Health Insurance Portability and Accountability Act regulations. An informed consent was obtained from the parents. The trial was registered at Clinical trials as NCT02126501.

\section{Study population}

One hundred and fifty four preterm infants born at the gestational age 26 weeks to 32 weeks requiring at least $48 \mathrm{~h}$ of NCPAP, admitted to the neonatal intensive care unit, met the eligibility criteria (Figure 1). All infants, including those who were initially intubated and then extubated to CPAP or those who were never intubated were eligible for the study. Those with chromosomal defects and severe congenital anomalies, including congenital heart disease, neurological malformations, chest wall or airway abnormalities and lung hypoplasia, were excluded from the study. A medical provider in the infant's circle of care approached their families to ask whether they would be willing to have a research coordinator speak to them about the study. When the family agreed, one of the study authors spoke with the family and described the study along with its risks and potential benefits. The parents or legal guardians were given adequate time to reflect on the information and have any questions answered before giving consent. Once consented, the principal investigator, who was not directly involved in the patient care, included the infant in the study and allocated the infant to a study group, utilizing the pre-prepared computergenerated randomization charts.

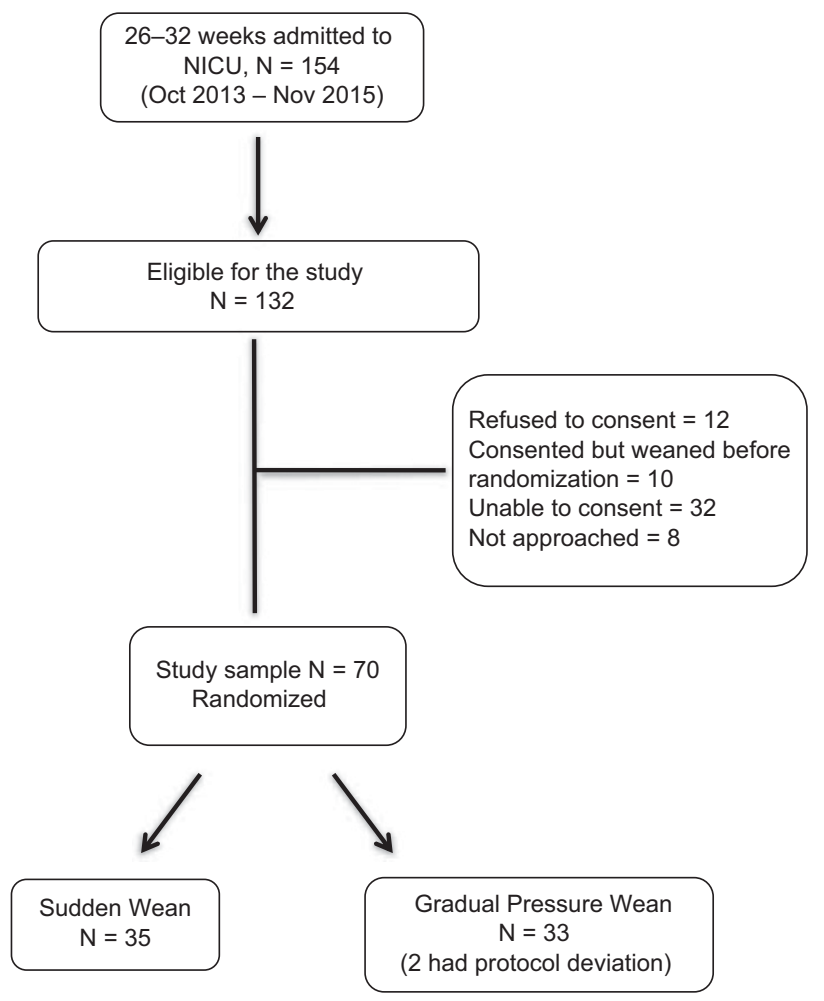

Figure 1. CONSORT flow diagram.
Study interventions

Application of NCPAP has been described earlier. ${ }^{23,26}$ Briefly, bubble NCPAP system (Fischer Paykel, Auckland, New Zealand) was used for pressure generation and the pressure in the circuit was checked by Criterion 40 pressure monitor (Respironics-Novametrix, Wallingford, CT, USA). NCPAP weaning was initiated as per the randomization allocation, when the infant was clinically stable on $\mathrm{FiO}_{2}$ of 0.21 and at $5 \mathrm{~cm}$ of $\mathrm{H}_{2} \mathrm{O}$ pressure for a minimum of $48 \mathrm{~h}$. Weaning was initiated when all other stability criteria (Table 1) were also met.

In the sudden weaning group, NCPAP at $\mathrm{FiO}_{2}$ of 0.21 and at $5 \mathrm{~cm}$ of $\mathrm{H}_{2} \mathrm{O}$ pressure was removed and kept off completely. In the gradual pressure wean group, the pressure was decreased by $1 \mathrm{~cm}$ of $\mathrm{H}_{2} \mathrm{O}$ pressure every $8 \mathrm{~h}$ from an initial positive end expiratory pressure (PEEP) of $5 \mathrm{~cm} \mathrm{H}_{2} \mathrm{O}$ until a PEEP of $3 \mathrm{~cm} \mathrm{H} \mathrm{H}_{2} \mathrm{O}$ was reached. NCPAP was then removed and kept off completely. In this group, the total time to wean off NCPAP to room air was $24 \mathrm{~h}$. The babies were not placed on any support such as NC after weaning. If the baby failed to wean on the initial attempt, repeated attempts were made in the original arm, with no crossover to the other arm after minimum of $24 \mathrm{~h}$ (Figure 2). Owing to the nature of NCPAP use in the neonatal intensive care unit, there was no blinding of the weaning process from the attending care teams, nursing staff, and study investigators.

Predetermined criteria (Table 2) were used to determine failure and success of the weaning trial. If the infant failed the trial, he/she would be placed back on $\mathrm{FiO}_{2}$ of 0.21 and $5 \mathrm{~cm}$ of $\mathrm{H}_{2} \mathrm{O}$ pressure. The next attempt at weaning was made after a minimum of $24 \mathrm{~h}$ and once the stability criteria were met again. Successful weaning was defined as the absence of persistent tachypnea, marked retractions or apneic episodes on room air without ventilator support, or need for supplemental oxygen for 7 days. The stability criteria, failure criteria and successful wean definition were available for the nursing staff as well as the clinical team at the bedside chart of the study patients.

\section{Study outcomes}

The primary outcome of interest was the success rate of the first trial of NCPAP weaning. Other outcomes of interest were the total number of days on NCPAP and the LOS in the hospital, and the weight and PMA at the following time points: starting NCPAP, reaching $\mathrm{FiO}_{2}$ of 0.21 , at first trial of weaning and at successful wean off NCPAP.

Additional clinical and demographic characteristics compared between the two groups include birth weight, gestational age, ethnicity and gender, and the presence of antenatal factors including the use of steroids, magnesium sulfate, chorioamnionitis (maternal fever of $>38^{\circ} \mathrm{C}$ and use of antibiotics), preeclampsia (blood pressure $>140 / 90 \mathrm{~mm} \mathrm{Hg}$ with proteinuria) and intrauterine growth retardation ( $<$ third percentile for weight). Postnatal factors including intubation, use of surfactant, use of caffeine, presence of patent ductus arteriosus (diagnosed by echocardiogram), sepsis (blood culture positive), anemia (hematocrit $<30 \%$ in the 7 days prior to weaning NCPAP), gastroesophageal reflux (diagnosed clinically with a response to $\mathrm{H}_{2}$ blockers) and the presence of intraventricular hemorrhage Stage 3 and 4 (diagnosed by ultrasound) were also compared between the two groups.

Sample size and statistical analysis

We estimated the sample size on the basis of the previous reported success in weaning from NCPAP in the first attempt, ${ }^{24}$ where the success rate of weaning from NCPAP during first attempt using sudden wean method was $33 \%$. We hypothesized that the gradual pressure wean method would be clinically important if associated with $66 \%$ success in weaning off NCPAP. To detect this difference, a sample size of 35 infants in each group provided $80 \%$ power with $a=0.05$. Continuous variables were analyzed using the Student's $t$-test and the categorical variables were analyzed using the $x^{2}$-test or Fisher's exact test, based on the distribution of the variables. All analyses were done on STATA version 14 (STATA, College Station, TX, USA).

\section{RESULTS}

Study population

Of the 154 infants admitted in the neonatal intensive care unit between October 2013 and November 2015,132 were eligible for the study. Of these, 70 babies were consented and randomized, but 2 of them were excluded due to protocol deviation (Figure 1). The 
Table 1. Criteria for readiness for weaning from NCPAP for $24-48 \mathrm{~h}$ before weaning

1. Continuous positive airway pressure (NCPAP) of $5 \mathrm{~cm} \mathrm{H}_{2} 0 ; 0.21 \mathrm{FiO}_{2}$

2. Normal work of breathing: no persistent tachypnea ( $>60$ breaths for $>2 \mathrm{~h}$ ), no marked retractions

3. No apnea (cessation of respiration $>20 \mathrm{~s}$ ) associated with bradycardia or cyanosis with $>2$ episodes in $12 \mathrm{~h}$ or $>3$ in $24 \mathrm{~h}$ with at least one

requiring bag and mask ventilation

4. Saturation $>90 \%$

5. Not currently treated for PDA or sepsis at the time of weaning

6. Tolerated time off NCPAP during nursing cluster care up to 15 min or more

Abbreviations: NCPAP, nasal continuous positive airway pressure; PDA, persistent ductus arteriosus.

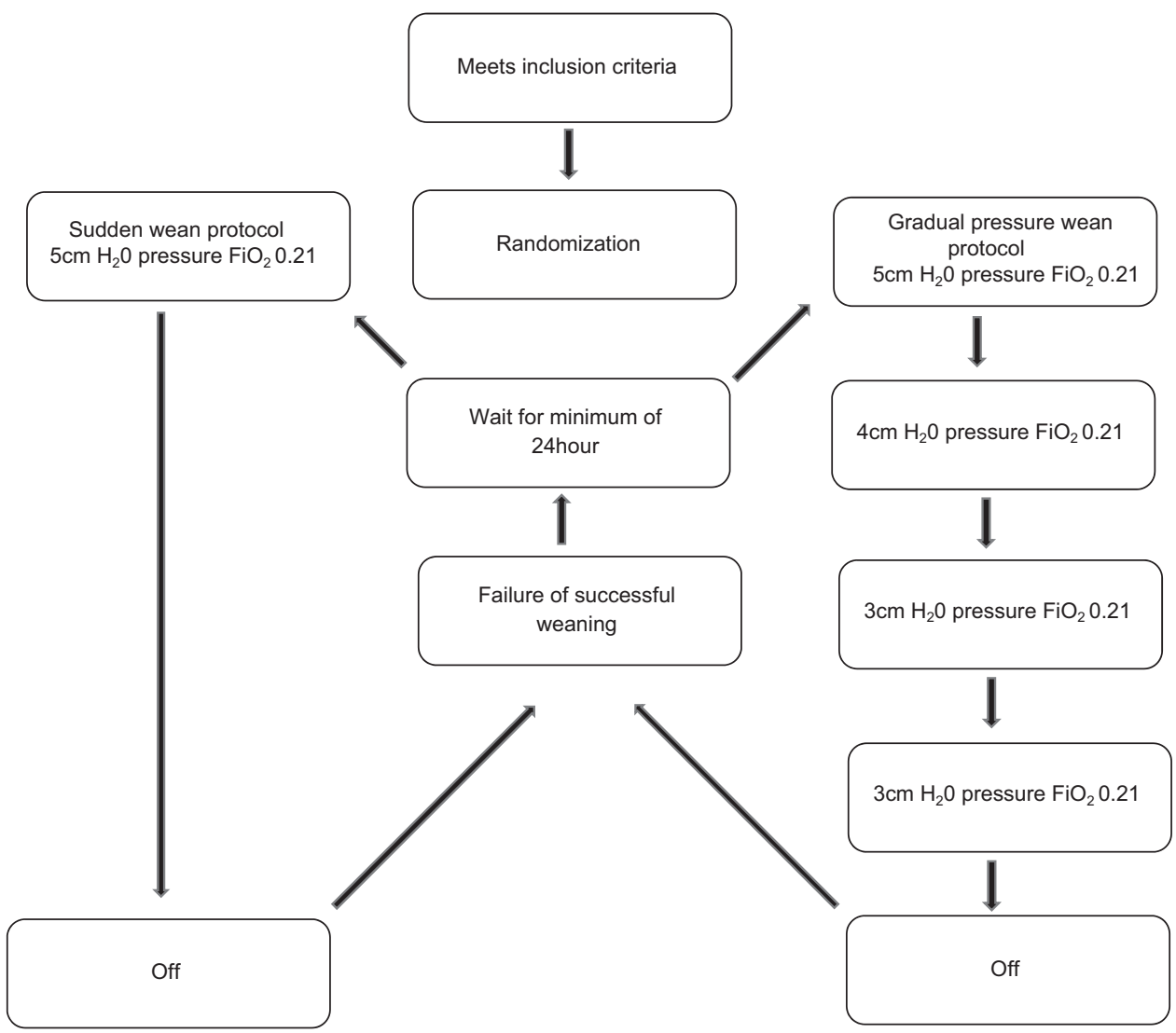

Figure 2. Details of study design.

Table 2. Criteria of failure of weaning from NCPAP present anytime till 7 days off NCPAP

1. Increased work of breathing: persistent tachypnea ( $>60$ for $>2 \mathrm{~h}$ ) and marked retractions

2. Apnea (cessation of respiration $>20 \mathrm{~s}$ ) associated with bradycardia or cyanosis with $>2$ episodes in $12 \mathrm{~h}$ or $>3$ in $24 \mathrm{~h}$ with at least one requiring

bag and mask ventilation

3. Oxygen requirement $>0.21$ to maintain the oxygen saturations $>90 \%$ for over $2 \mathrm{~h}$ in $24 \mathrm{~h}$

4. Abnormal blood gases ( 2 arterial samples $>2 \mathrm{~h}$ apart) with low $\mathrm{pH}<7.2, \mathrm{PaO}_{2}>65 \mathrm{~mm} \mathrm{Hg}, \mathrm{PaO}_{2}<50 \mathrm{~mm} \mathrm{Hg}$

Abbreviations: NCPAP, nasal continuous positive airway pressure.

clinical characteristics did not differ between the sudden wean group and the gradual wean group though use of prenatal steroids, which was higher $(P=0.02)$ in the gradual wean group (Table 3 ). Logistic regression analysis on the association of weaning method with success at the first attempt, adjusting for use of antenatal steroids was performed. The antenatal steroids were not independently associated with success of first attempt among those who underwent gradual wean $(\beta=-7.36,(-26.73$ to $12.02, P=0.45))$. The three episodes of sepsis, two sudden group and one in gradual group were late-onset sepsis and grew Staphylococcus epidermidis.

Comparison of two weaning methods

More infants in the gradual wean group were successfully weaned off NCPAP as compared with the sudden wean group (22 vs 14 , $P=0.03$ ). The median number of attempts required to be successfully off NCPAP ranged from 1 to 5 and did not differ 
Table 3. Comparison of clinical characteristics of the two study groups $n(\%)$

\begin{tabular}{|c|c|c|c|}
\hline Parameter & $\begin{array}{l}\text { Sudden wean } \\
\qquad \mathrm{n}=35\end{array}$ & $\begin{array}{l}\text { Gradual wean } \\
\qquad \mathrm{n}=33\end{array}$ & P-values \\
\hline $\begin{array}{l}\text { Weight in gram } \\
\text { (mean } \pm \text { s.d.) }\end{array}$ & $1305 \pm 374$ & $1198 \pm 330$ & 0.22 \\
\hline GA in weeks (mean \pm s.d.) & $28.7 \pm 1.8$ & $28.5 \pm 1.9$ & 0.75 \\
\hline Gender M:F & $3: 2(21 / 14)$ & $7: 4(21 / 12)$ & 0.76 \\
\hline Ethnicity & & & 0.88 \\
\hline White & $8(22.8)$ & $10(30.3)$ & \\
\hline African American & $6(17.1)$ & $6(18.1)$ & \\
\hline Hispanic & $4(11.4)$ & $4(12.1$ & \\
\hline Asian & $17(48.5)$ & $13(39.4)$ & \\
\hline Antenatal steroids & $29(82.8)$ & $33(100.0)$ & 0.02 \\
\hline Chorioamnionitis & $2(5.7)$ & $2(6.0)$ & 1 \\
\hline IUGR & $2(5.7)$ & $4(12.1)$ & 0.42 \\
\hline Intubation & $14(40.0)$ & 7 (21.2) & 0.09 \\
\hline $\begin{array}{l}\text { Days on ventilator } \\
\text { (mean } \pm \text { s.d.) }\end{array}$ & $6.13 \pm 2.64$ & $8.28 \pm 8.84$ & 0.48 \\
\hline Surfactant & $7(20.0)$ & $6(18.2)$ & 0.85 \\
\hline $\begin{array}{l}\text { Days to reach RA NCPAP } \\
\text { (mean } \pm \text { s.d.) }\end{array}$ & $5.6 \pm 12.4$ & $5.7 \pm 12.6$ & 0.48 \\
\hline Anemia & $30(85.7)$ & $29(87.9)$ & 1 \\
\hline Caffeine use & $26(74.2)$ & $22(66.7)$ & 0.49 \\
\hline IVH & 4 (11.4) & $3(9.1)$ & 1 \\
\hline Sepsis & $2(5.7)$ & $1(3.0)$ & 1 \\
\hline PDA & $22(62.8)$ & $16(48.5)$ & 0.23 \\
\hline GERD & $5(14.3)$ & $2(6.0)$ & 0.43 \\
\hline BPD & $3(8.6)$ & $3(9.1)$ & 1 \\
\hline \multicolumn{4}{|c|}{$\begin{array}{l}\text { Abbreviations: BPD, bronchopulmonary disease; F, female; GA, gestational } \\
\text { age; GERD, gastro-esophageal reflux disease; IUGR, intra-uterine growth } \\
\text { retardation; IVH, intraventricular hemorrhage; M, male; PDA, persistent } \\
\text { ductus arteriosus; RA, room air. }\end{array}$} \\
\hline
\end{tabular}

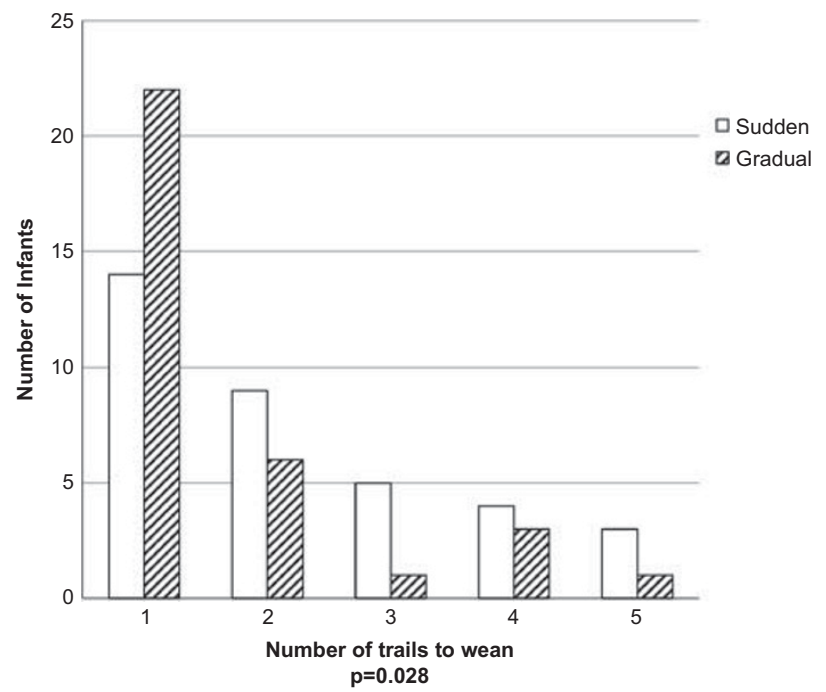

Figure 3. Number of trials to successful weaning of NCPAP by sudden and gradual pressure weaning methods.

between the two groups $(P=0.2)$, (Figure 3$)$. There was no difference in the weight and the PMA between the sudden wean and the gradual pressure wean group at the various study time points (Figures $4 a$ and b). The gestational age when infants came off the supplemental oxygen did not differ between the sudden and gradual wean $(29.3 \pm 1.9$ vs $29.6 \pm 2.8$ weeks, $P=0.57)$. Similarly, the weight at wean off supplemental $\mathrm{O}_{2}$ did not differ

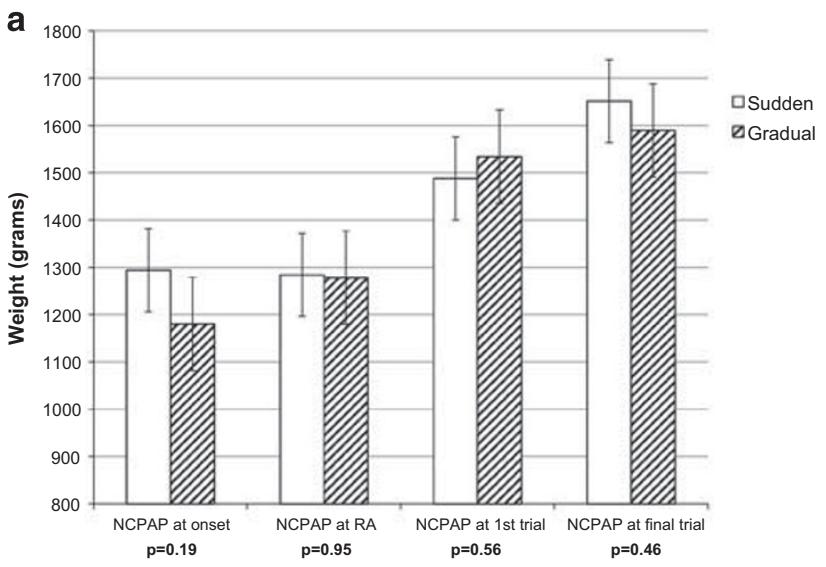

b

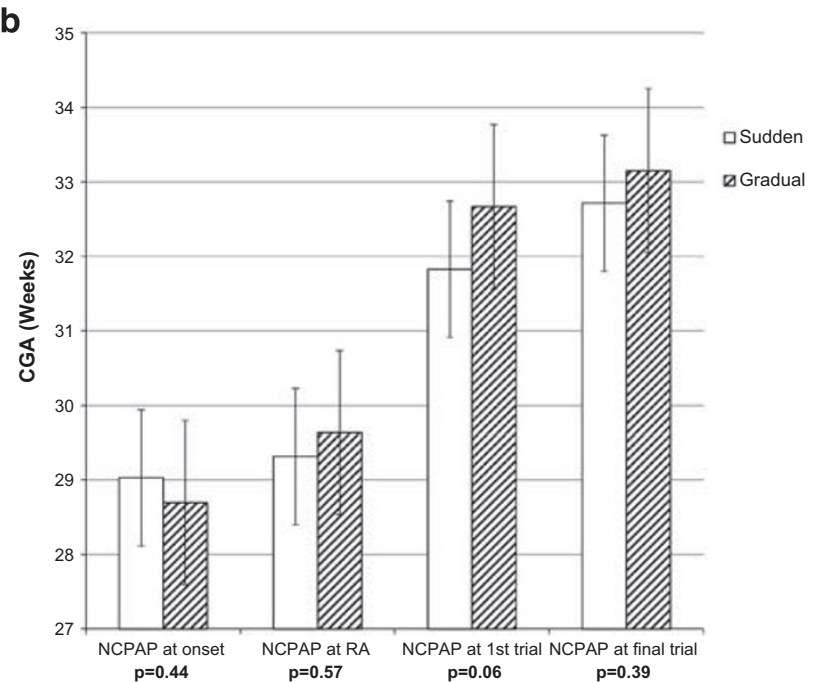

Figure 4. (a) Comparison of weight at various study time points between the sudden and gradual pressure wean groups. (b) Comparison of corrected gestational age (CGA) at various study time points between the sudden and gradual pressure wean groups.

between sudden and gradual wean $(1284 \pm 372 \mathrm{~g}$ vs $1278 \pm 474 \mathrm{~g}$, $P=0.95)$. The infants were successfully weaned at $32.7 \pm 1.8$ weeks versus $33.1 \pm 2.4$ weeks $(P=0.39)$ and at $1651 \pm 291 \mathrm{~g}$ versus $1589 \pm 398 \mathrm{~g}(P=0.46)$, in the sudden wean and gradual wean groups, respectively. The LOS did not differ between the sudden wean $(62.9 \pm 25.4$ days) and gradual wean group (62.9 \pm 22.1 days) $(P=0.99)$. The total days on NCPAP in the sudden wean group $(27.4 \pm 19.3$ days) did not differ from the gradual wean group (32.0 \pm 23.7 days) $(P=0.38)$.

Comparison between those included in the trial and those who met the criteria but were not enrolled in the trial

The 62 infants who were eligible, but were not included in the trial, were found to have a higher gestational age as compared with the 70 infants who were included in the trial $(29.3 \pm 1.5$ versus $28.6 \pm 1.8$ weeks, $P=0.01$ ). Although the LOS was also significantly less among those not included in the trial as compared with those included in the trial $(53.4 \pm 2.9$ vs $62.9 \pm 2.8$ days, $P=0.02)$, this difference was not significant when corrected for gestational age. There was an increased incidence of bronchopulmonary disease $(P=0.03)$ in those not included in the trial as compared with those included in the trial despite being significantly more mature at birth. There was no difference $(P=0.27)$ in the time spent of NCPAP in both the groups (Table 4). 
Table 4. Comparison of clinical characteristics of the neonates included in the study and those infants who met inclusion criteria but not enrolled in the study $n(\%)$

\begin{tabular}{|c|c|c|c|}
\hline Parameter & $\begin{array}{c}\text { Nonrandomized } \\
n=62\end{array}$ & $\begin{array}{c}\text { Randomized } \\
\mathrm{n}=70\end{array}$ & P-values \\
\hline Weight mean \pm s.d. (g) & $1343 \pm 325$ & $1254 \pm 355$ & 0.14 \\
\hline GA mean \pm s.d. (weeks) & $29.4 \pm 1.5$ & $28.6 \pm 1.8$ & 0.01 \\
\hline Gender M:F & 24 & 42 & 0.01 \\
\hline Ethnicity & & & 0.10 \\
\hline White & $27(43.5)$ & 18(25.7) & \\
\hline African American & $8(12.9)$ & $12(17.1)$ & \\
\hline Hispanic & $10(16.1)$ & $8(11.4)$ & \\
\hline Asian & $17(27.4)$ & $30(42.8)$ & \\
\hline Antenatal steroids & $49(79.0)$ & $62(88.6)$ & 0.05 \\
\hline Chorioamnionitis & $1(2.0)$ & $4(5.7)$ & 0.21 \\
\hline IUGR & $5(8.1)$ & $6(8.6)$ & 0.88 \\
\hline Intubation & $12(19.3)$ & $21(30.0)$ & 0.13 \\
\hline Surfactant & $8(12.9)$ & $13(18.6)$ & 0.33 \\
\hline Anemia & 57 (91.9) & $59(84.3)$ & 0.34 \\
\hline Caffeine use & $35(56.4)$ & $48(68.6)$ & 0.09 \\
\hline IVH & $8(12.9)$ & 7 (10.0) & 0.64 \\
\hline Sepsis & $9(14.5)$ & $3(4.3)$ & 0.05 \\
\hline PDA & $26(41.9)$ & $38(54.3)$ & 0.11 \\
\hline GERD & $5(8.0)$ & $7(10.0)$ & 0.66 \\
\hline BPD & $14(22.6)$ & $6(8.6)$ & 0.03 \\
\hline
\end{tabular}

\section{DISCUSSION}

In a randomized control trial, we demonstrated that preterm infants born at 26 to 32 weeks gestation, who required NCPAP, had higher success rate at the first attempt to wean from NCPAP by gradual pressure weaning off NCPAP as compared with the sudden weaning method. There were no differences in the gestational age and weight at the time of successful wean, the total duration of oxygen use or time on NCPAP and the length of hospital stay between the two weaning methods. These findings suggest that gradual pressure wean may be associated with greater success of weaning from NCPAP.

Our findings concur with previous studies that used the gradual pressure weaning method. ${ }^{19,20}$ Singh et al. ${ }^{19}$ demonstrated that time on NCPAP was significantly less when weaning was by gradual decrease in pressure as compared with increasing time off from NCPAP. However, the authors did not comment on the success of the first trial as compared with successive attempts at weaning from NCPAP. Similarly, Soe ${ }^{20}$ reported no significant difference in the number of NCPAP days but had a trend towards shorter time on weaning with gradual decrease in pressure. However, the details on the NCPAP delivery method, need for NC or criteria for readiness, failure and the methods used for weaning were not reported in either of the studies. Further, the time on NCPAP in both these studies was short, ranging from 6 to 15 days, which could be indicative of less severe respiratory distress during hospital stay, as compared with our study where the time on NCPAP was 3 to 4 weeks, before attempts were made to wean off NCPAP. In contrast to these previous two studies, we used welldefined and stringent criteria to define the readiness for weaning, as well as the success and failure of weaning. Furthermore, although these investigators described success of weaning as being off NCPAP for variable periods off CPAP, we had observed that infants who may have been initially stable on room air for $24 \mathrm{~h}$ could still fail after a few days and may require to be placed back on NCPAP. ${ }^{23,24}$ For these reasons, our successful wean criteria was more stringent, as it was defined as NCPAP being off for longer period of 7 days. We also clearly and extensively describe details of NCPAP delivery, having used a method that has been shown to be one of the most commonly used and cost-effective methods to deliver NCPAP. ${ }^{26}$

Gradual decrease in pressure was more successful in the first attempt off NCPAP in our study and could be related to the prevention of atelectasis by gradually weaning pressure as compared with sudden weaning. Appropriate level of PEEP for preterm infants depends on factors influencing the underlying pathophysiology; however, gradual increase in functional residual capacity is noted from pressure of 2 to $5 \mathrm{~cm} \mathrm{H}_{2} \mathrm{O}$ with the normal values of healthy term infants being achieved at $5 \mathrm{~cm} \mathrm{H}_{2} \mathrm{O} .{ }^{27}$ Thus, 3 to $5 \mathrm{~cm}$ of $\mathrm{H}_{2} \mathrm{O}$ pressure in the study may not generate adequate PEEP in each infant depending on the pathophysiology of the disease; hence, further studies on weaning should be stratified by gestational age and disease entity.

Using other methods for weaning from NCPAP, Todd et al. ${ }^{22}$ in a multicenter trial found that sudden weaning was better than weaning by cycling time off and was associated with less time on oxygen and shorter LOS in the hospital. We had studied this methodology and had not observed any differences between these two methods of weaning from NCPAP. ${ }^{23}$ The difference observed by Todd et al. ${ }^{22}$ could be related to higher number of preterm infants and higher incidence of patent ductus arteriosus in the gradual time-off group in the multicenter trial. ${ }^{28}$ Further, instead of weaning from NCPAP to room air, studies have compared NCPAP weaning with weaning to $\mathrm{NC}^{29}$ or high flow $\mathrm{NC}^{30}$ Weaning to NC or high flow NC are associated with longer duration of oxygen exposure and respiratory support, and there is variable amount of residual PEEP, provided if NC is used with higher flows, ${ }^{31}$ which may be similar to gradual pressure wean without the accurate measure of PEEP. Moreover, all the studies have lower total number of days on NCPAP as compared with our study at the time of initiation of weaning, suggesting that NCPAP may have been used after extubation, rather than as a primary mode of ventilation, as was the case in our study. The babies who initially failed on NCPAP were intubated (about 30\% of infants included in the study) and the rest were managed only on NCPAP, and hence a longer duration on NCPAP in our study as compared with previously reported studies. This difference in duration on CPAP highlights that the babies in previous studies may also have had less severe respiratory disease or used CPAP during post extubation period.

There was no difference in the duration on NCPAP between the infants included in the study as compared with those who met the inclusion criteria but were not included in the study. However, the latter had significantly higher incidence of bronchopulmonary disease, despite being significantly more mature. Our findings suggest that having a protocol for readiness for weaning may also help in taking neonates off the NCPAP appropriately and may impact the incidence of bronchopulmonary disease.

Given the nature of the study, there are limitations in our study, most important being the inability to blind the providers to intervention. However, the possible impact of these lack of blinding on outcomes was decreased by utilization of strict criteria for weaning. Another disadvantage to the gradual pressure weaning method is that the protocol is inherently longer than that of sudden weaning method. This differences in the length of weaning between the two study groups are important as with failure of weaning of NCPAP, next trial of weaning could only occur after $24 \mathrm{~h}$ when the stability criteria were met; hence, more trials of sudden wean could be performed compared with the gradual group and could have an impact on the length on NCPAP. We did have a shorter gradual pressure weaning protocol as compared with earlier studies. ${ }^{19,20,22}$ This helped to minimize difference in the number of trials between the two methods. The 
optimal length of gradual pressure weaning method needs to be studied further. Furthermore, utilization of strict criteria for weaning improved the success from the weaning from 33\% from historical data to $40 \%$ observed in this study; hence, the sample size calculated at the start of the trial was not large enough to show any differences between the two weaning methods, especially in number of trials, time on NCPAP and oxygen, and the length of stay.

We conclude that gradual pressure weaning of NCPAP was more successful in weaning off NCPAP in the first trial, but was not different in the number of attempts to wean off NCPAP, time on NCPAP and oxygen, and the length of stay when compared with sudden weaning. Our findings may be useful to develop guidelines for the readiness of weaning NCPAP and defining successful wean and failure of weaning from NCPAP. These may have an impact on the time on NCPAP and decrease inter-provider variability that is inherent to ad-hoc weaning. Future multicenter trials are needed for stratification by gestational age and by underlying pulmonary morbidity for validation and generalizability of our findings.

\section{COLLABORATORS OF MAIMONIDES NEONATAL GROUP}

Michele Dyan, Panayot Filipov, Anju Gupta, Tania Mangones and Melissa Tsai.

\section{CONFLICT OF INTEREST}

The authors declare no conflict of interest.

\section{ACKNOWLEDGEMENTS}

Trial Registration: ClinicalTrials.gov Identifier: NCT02126501

\section{REFERENCES}

1 De Paoli AG, Morley C, Davis PG. Nasal NCPAP for neonates: what do we know in 2003? Arch Dis Child Fetal Neonatal Ed 2003; 88: F168-F172.

2 Morley CJ, Davis PG, Doyle LW, Brion LP, Hascoet JM, Carlin JB et al. Nasal NCPAP or intubation at birth for very preterm infants. N Engl J Med 2008; 358: 700-708.

3 Aly $\mathrm{H}$. Ventilation without tracheal intubation. Pediatrics 2009; 124: 786-789.

4 Dunn MS, Kaempf J, de Klerk A, de Klerk R, Reilly M, Howard D et al. Randomized trial comparing 3 approaches to the initial respiratory management of preterm neonates. Pediatrics 2011; 128(5): e1069-e1076.

5 Schmolzer GM, Kumar M, Pichler G, Aziz K, O'Reilly M, Cheung PY. Non-invasive versus invasive respiratory support in preterm infants at birth: systematic review and meta-analysis. BMJ 2013; 347: 5980.

6 Pérez LA, González DM, Álvarez, Karen Margarita de Jesús, Díaz-Martínez LA. Nasal NCPAP versus mechanical ventilation in 28 to 32 -week preterm infants with early surfactant administration. Biomédica 2014; 34(4): 612-623.

7 Gregory GA, Kitterman JA, Phibbs RH, Tooley WH, Hamilton WK. Treatment of the idiopathic respiratory-distress syndromewith continuous positive airway pressure. N Engl J Med 1971; 284: 1333-1340.

8 Avery ME, Tooley WH, Keller JB, Hurd SS, Bryan MH, Cotton RB et al. Is chronic lung disease in low birth weight infants preventable? A survey of eight centers. Pediatrics 1987; 79: 26-30.

9 Friedman CA, Menchaca RC, Baker MC, Rivas CK, Laberge RN, Rios EH et al. Bubble nasal NCPAP, early surfactant treatment, and rapid extubation are associated with decreased incidence of bronchopulmonary dysplasia in very-low-birth-weight newborns: efficacy and safety considerations. Respir Care 2013; 58(7): 1134-1142.

10 Narendran V, Donovan EF, Hoath SB, Akinbi HT, Steichen JJ, Jobe AH. Early bubble CPAP and outcomes in ELBW preterm infants. J Perinatol. 2003; 23: 195-199.

11 De Klerk AM, De Klerk RK. Nasal continuous positive airway pressure and outcomes of preterm infants. J Paediatr Child Health 2001; 37: 161-167.

12 Zhang S, Garbutt V, McBride JT. Strain-induced growth of the immature lung J Appl Physiol (1985) 1996; 81(4): 1471-1476.

13 Newnam KM, McGrath JM, Estes T, Jallo N, Salyer J, Bass WT. An integrative review of skin breakdown in the preterm infant associated with nasal continuous positive airway pressure. J Obstet Gynecol Neonat Nursing 2013; 42(5): 508-516.

14 Levene Ml, Fawer CL, Lamont RF. Risk factors in the development of intraventricular haemorrhage in the preterm neonate. Arch Dis Child 1982; 57: 410-417.

15 Aly $\mathrm{H}$, Hammad TA, Essers J, Wung JT. Is mechanical ventilation associated with intraventricular hemorrhage in preterm infants? Brain Dev 2012; 34: 201-205.

16 Jardine L, Davies MW. Withdrawal of neonatal continuous positive airway pressure: current practice in Australia. Pediatr Int 2008; 50: 572-575.

17 Amatya S, Rastogi D, Bhutada A, Rastogi S. Weaning of nasal CPAP in preterm infants: who, when and how? a systematic review of the literature. World J Pediatr 2015; 11(1): 7-13.

18 Abdel-Hady H, Mohareb S, Khashaba M, Abu-Alkhair M, Greisen G. Randomized controlled trial of discontinuation of nasal-NCPAP in stable preterm infants breathing room air. Acta Paediatr 1998; 87: 82-87.

19 Singh SD, Bowe L, Clark P, Glover K, Pasquill A, Robinson NMJ et al. Is decreasing pressure or increasing time of a better strategy in weaning VLBW infants from NCPAP. Eur J Paediatr 2006; 165(Suppl): 48.

20 Soe A. Weaning from nasal NCPAP in premature infants. Inspire 2007; 5: 8-10.

21 Jardine LA, Inglis GD, Davies MW. Strategies for the withdrawal of nasal continuous positive airway pressure (NCPAP) in preterm infants. Cochrane Database Syst Rev 2011; 2: CD006979.

22 Todd DA, Wright A, Broom M, Chauhan M, Meskell S, Cameron C et al. Methods of weaning preterm babies $<30$ weeks gestation off NCPAP: a multicentre randomised controlled trial. Arch Dis Child Fetal Neonatal Ed 2012; 97: F236-F240.

23 Rastogi S, Wong W, Gupta A, Bhutada A, Rastogi Deepa, Maimonides Neonatal Group. Gradual versus sudden weaning from nasal NCPAP in preterm infants: a pilot randomized controlled trial. Respir Care 2013; 58: 511-516.

24 Rastogi S, Rajasekhar H, Gupta A, Bhutada A, Rastogi D, Wung JT. Factors affecting the weaning from nasal NCPAP in preterm neonates. Int J Pediatr 2012; 2012: 416073.

25 Bloom M, Ying L, Wright A, Stewart A, Abdel-Latif ME, Shadbolt B et al. Ceasing NCPAP At standarD criteriA (CICADA): impact on weight gain, time to full feeds and caffeine use. Arch Dis Child Fetal Neonatal Ed 2014; 99: F423-F425.

26 Polin RA, Sahni R. Newer experience with NCPAP. Semin Neonatol 2002; 7: 379-389.

27 Bamat N, Millar D, Suh S, Kirpalani H. Positive end expiratory pressure for preterm infants requiring conventional mechanical ventilation for respiratory distress syndrome or bronchopulmonary dysplasia. Cochrane Database Syst Rev 2012; 1 Art. No.: CD004500.

28 Yin Y, Broom M, Wright A, Hovey D, Abdel-Latif ME, Shadbolt B et al. Ceasing NCPAP At standard criteriA ( CICADA): predicting successful outcome. Eur J Pediatr 2016; 175: 81-87.

29 Abdel-Hady H, Shouman B, Aly H. Early weaning from NCPAP to high flow nasal cannula in preterm infants is associated with prolonged oxygen requirement: a randomized controlled trial. Early Hum Dev 2011; 87: 205-208.

30 Tang J, Reid S, Lutz T, Malcolm G, Oliver S, Osborn DA. Randomised controlled trial of weaning strategies for preterm infants on nasal continuous positive airway pressure. BMC Pediatr 2015; 15(1): 1.

31 Spence KL, Murphy D, Kilan C, McGonigle R, Kilani RA. High-flow nasal cannula as a device to provide continuous positive airway pressure in infants. $J$ Perinatol 2007; 27(12): 772-775. 